\title{
Interaction of Peptides Containing CRAC Motifs with Lipids in Membranes of Various Composition
}

\author{
P. E. Volynsky ${ }^{a}$, T. R. Galimzyanov ${ }^{b}$, and S. A. Akimov ${ }^{b, *}$ \\ ${ }^{a}$ Shemyakin-Ovchinnikov Institute of Bioorganic Chemistry, Russian Academy of Sciences, Moscow, 117997 Russia \\ ${ }^{b}$ Frumkin Institute of Physical Chemistry and Electrochemistry, Russian Academy of Sciences, Moscow, 119071 Russia \\ *e-mail: akimov_sergey@mail.ru
}

Received September 17, 2020; revised September 21, 2020; accepted September 22, 2020

\begin{abstract}
The lateral distribution of integral and peripheral proteins, as well as lipids in the plasma membranes of mammalian cells is extremely heterogeneous. It is believed that various lipid-protein domains are formed in membranes. Domains enriched in sphingomyelin and cholesterol are called rafts. It is assumed that the distribution of proteins into rafts is largely related to the presence in their primary sequence of a specific amino acid region called the CRAC motif, which is responsible for cholesterol binding. In this work, the interaction of two peptides containing CRAC motifs in their structure with membranes of different compositions was studied by means of molecular dynamics. It has been shown that the average number of lipid molecules in contact with each peptide is proportional to the mole fraction of lipid in the membrane. The predominant interaction of peptides with cholesterol was not observed. In addition, cholesterol did not form long-lived contacts with any amino acid or amino acid sequence. We suppose that in some cases the predominant lateral distribution of peptides and proteins containing CRAC motifs into rafts may be due to amphipathicity of the CRAC motif rather than due to specific strong binding of cholesterol.
\end{abstract}

Keywords: lipid membrane, ordered domains, CRAC motif, molecular dynamics, amphipathic peptide DOI: $10.1134 /$ S1990747821010074

\section{INTRODUCTION}

Lateral distribution of integral and peripheral proteins in plasma membranes is extremely heterogeneous $[1,2]$. It has been shown that various proteins form clusters of 10-200 nm in size [1]. There are at least two non-overlapping types of such clusters, differing in the composition of the proteins that form them [3]. In other words, some of the membrane proteins are always located in clusters of the first type, and the other part is always in clusters of the second type, and the proteins that form the first type of clusters are almost never co-localized with the proteins that form the second type of clusters. When cell membranes are treated with a soft non-ionic detergent in low concentration at a low temperature $\left(\sim 4^{\circ} \mathrm{C}\right)$ and subsequently centrifuged, membrane proteins are separated into two fractions corresponding to two types of clusters [4]. In addition to the difference in protein composition, the fractions also differ in lipid content: sphingomyelin is almost completely distributed into the lowdensity fraction, while the high-density fraction is enriched in unsaturated lipids [3, 4]. Cholesterol, according to different data, is distributed either almost evenly between the fractions [3,5] or has some preference for the low-density fraction [4]. It is assumed that protein clusters in plasma membranes are actually lipid-protein domains, the compositions of which approximately coincide with the compositions of the fractions obtained by centrifugation; the domains enriched with sphingomyelin and cholesterol are called rafts [6]. The great interest in the study of rafts is conditioned by that they are involved in processes catalyzed by membrane proteins, and the presence of a raft environment is critical for the proper functioning of proteins $[2,7,8]$.

The small size of the domains significantly complicates their study in cell membranes. In model purely lipid membranes, the composition of which is close to the composition of the outer monolayer of plasma membranes, phase separation could be induced by decreasing temperature. The separation results in the formation of micron-sized domains that can be studied by various experimental methods, including optical [3, 5, 9-12]. Similar to rafts, such domains are also relatively resistant to detergent action and are enriched in sphingomyelin and cholesterol; they are used as a model of cellular rafts $[5,13]$. In model membranes, it was found that the lipid in the domains is in a liquidordered $\left(\mathrm{L}_{\mathrm{o}}\right)$ state, while the surrounding membrane is in a liquid-disordered $\left(\mathrm{L}_{\mathrm{d}}\right)$ state [10-12]. Due to the difference in ordering, the thickness of the domain bilayer is greater than the thickness of the surrounding 
membrane [13]. Domains are characterized by relatively high elastic moduli [14], and the diffusion coefficient is lowered in them [10].

Clustering of various proteins and lipids in a limited area of the plasma membrane is necessary for the normal course of vital cellular processes, in particular, membrane fusion and fission [15, 16], the transmission of cellular signals $[17,18]$, etc. In addition to normal physiological processes, such an organization of lipid-protein platforms is a key stage in pathological processes: the assembly of new viral particles on the membranes of a cell infected with enveloped viruses. It has been shown experimentally that the lipid composition of the membrane of influenza and immunodeficiency viruses practically coincides with the lipid composition of the raft phase of the cell membrane [19]. It is assumed that the assembly of new virions occurs on the domains of the liquid-ordered phase, into which viral membrane proteins should be predominantly distributed. Thus, these proteins should have a pronounced affinity for rafts.

A number of studies suggest that the affinity of various proteins for cholesterol-rich plasma membrane domains can be provided by the presence in their structure of specific sequences of amino acid residues, presumably "recognizing" and binding cholesterol [20], called "CRAC" - cholesterol recognition amino acid consensus [21]. CRAC motifs are described by the general formula $-\mathrm{L} / \mathrm{V}-(\mathrm{X})(1-5)-\mathrm{Y} / \mathrm{W}-(\mathrm{X})(1-5)-\mathrm{R} / \mathrm{K}-$, where $(X)(1-5)$ denotes from one to five residues of arbitrary amino acids, symbol "/" means "or". Such sequences have been found in the primary structure of various proteins distributed into the raft phase of cell membranes, including caveolin, membrane receptors $[20,22]$, as well as a fusion protein (hemagglutinin) [23] and matrix protein (M1) of influenza virus [24]. In [25], it was shown that synthetic CRAC-containing peptides corresponding to the 3rd, 6th, and 13th $\alpha$-helices of M1 protein, to varying degrees modulate the cholesterol-dependent interaction of cultured IC-21 macrophages with $2-\mu \mathrm{m}$ particles. In similar experiments, it was found that peptide RTKLWEMLVELGNMDKAVKLWRKLKR-NH $\mathrm{N}_{2}$ containing two CRAC motifs from the 3rd and 6th $\alpha$-helices of M1 protein, significantly affects the activity of macrophages IC-21 in a complex manner [26].

In [20] it was suggested that along with post-translational lipidation of proteins, the presence of amino acid sequences in their structure that bind certain lipid components can determine the distribution of proteins into membrane domains enriched with such components. The CRAC motif was considered as such an amino acid sequence by the example of caveolin- 1 and caveolin-2, for which it was shown that palmitoylation is not a necessary condition for their distribution into caveolae, whose membrane is in a raft state [2]. At the same time, caveolin-3 does not contain CRAC motifs; nevertheless, it is distributed into rafts, apparently due to post-translational palmitoylation [20]. Based on the experimental data obtained in [23], a hypothesis was put forward about the essential role of the CRAC motif in the distribution of hemagglutinin, a fusion protein of influenza virus, into the liquid-ordered phase of the membrane. However, the experimentally observed "contrast" in the distribution of cholesterol over the plasma membrane is, as a rule, significantly lower than that of proteins associated with rafts [3, 27]. At the same time, the cellular domains of the liquidordered phase are significantly enriched in sphingomyelin. This suggests that CRAC motifs may also interact with sphingomyelin, or the specific interaction of CRAC motifs with cholesterol may depend on the presence of sphingomyelin or other lipids. As far as we know, these assumptions have not been systematically tested.

In the present work, the interaction of peptides containing CRAC motifs in their structure with lipid molecules in membranes of various compositions was studied by molecular dynamics (MD) methods. The modeling performed did not allow us to reveal an increased affinity for cholesterol in the studied peptides, regardless of the presence of sphingomyelin in the system. In all cases, 1-2 cholesterol molecules interacted with peptides, which corresponded to the average concentration of this lipid in the system. Similar results were obtained for the interaction of peptides with sphingomyelin. The study of the localization of cholesterol interacting with the peptide also failed to reveal stable binding sites: cholesterol interacted with residues oriented into the membrane and did not form long-lived complexes with the peptide. The results obtained allow us to conclude that the presence of CRAC motif in the structure of a peptide by itself is not a universal criterion for strong specific binding of cholesterol by this peptide.

\section{MATERIALS AND METHODS}

The regions of the third and sixth amphipathic helices of the matrix protein $\mathrm{M} 1$ of influenza virus were used as test peptides: peptide $\mathrm{p}_{1}=$ LEVLMEWLKTR, peptide $\mathrm{p}_{2}=$ NNMDKAVKLYRKLKR (amino acids forming the CRAC motif are shown in bold), first described in [25] as peptides containing CRAC motives and characterized by their effect on cholesterol-dependent particle binding by macrophages IC-21. For each of these peptides, MD simulations of a length of $10 \mu$ s were carried out in the presence of lipid bilayers of the following compositions: (1) dioleoylphosphatidylcholine (DOPC), 200 lipids, system designation-DOPC; (2) DOPC/cholesterol (Chol), 180 DOPC molecules, 20 cholesterol molecules, system designationDOPC/Chol; (3) DOPC/dipalmitoylsphingomyelin (DPSM), 180 DOPC molecules, 20 dipalmitoylsphingomyelin molecules, system designation-DOPC/DPSM; (4) DOPC/DPSM/Chol, 160 DOPC molecules, 
20 DPSM molecules, 20 Chol molecules, system designation-DOPC/DPSM/Chol.

The affinity of peptides to various lipids was studied by the molecular dynamics method in a coarsegrained approximation. The calculations were carried out using the Gromacs 2016.3 program in the martini2.2P force field for calculations with polarized water [28]. For the calculations, we used NVidia GeForce GTX 1080 video cards (AsusTek Computer Inc., Taiwan), which allow calculating approximately $0.5 \mu$ s of the trajectory per day. For the system of each composition, one trajectory of a length of $10 \mu$ s was calculated. Molecular topologies of lipids (DOPC, DPSM, Chol) were taken from the available lipid database [29]. MD simulations were performed at a constant temperature $(295 \mathrm{~K})$ maintained using the NoseHoover thermostat [30, 31]. During the calculation, the pressure was maintained (semi-isotropic tuning scheme) using the Parinello-Raman barostat [32, 33]. The integration step in the MD process was $10 \mathrm{fs}$. When taking into account long-range interactions, we used the parameters recommended for the selected force field: van der Waals interactions-the shift scheme with radii $0.9 / 1.2 \mathrm{~nm}$, electrostatic interactions-the shift scheme with radii $0.0 / 1.2 \mathrm{~nm}$. The duration of the calculated trajectories was $10 \mu \mathrm{s}$. Electrostatic interactions were calculated using the dielectric constant of the membrane medium $\varepsilon=2.5$. The systems under study consisted of 200 lipid molecules and approximately 20000 water molecules. At the first stage, calculations of the equilibrium states of bilayers were carried out. In the starting configuration of each monolayer, lipids were randomly distributed on a twodimensional grid with a step of $0.8 \mathrm{~nm}$. Two assembled monolayers were combined into a bilayer so that the distance between model particles, including phosphorus atoms, of different monolayers along the normal to the bilayer was $3.4 \mathrm{~nm}$. Water was added to the resulting system. The model system was balanced for $100 \mathrm{~ns}$. At the second stage, a peptide was added to the aqueous part of the system. After the initial equilibration of the system, at which the energy was minimized during $100 \mathrm{~ns}$ at constant pressure (semi-isotropic tuning scheme) using the Berendsen barostat [34], the main calculation of the MD was started.

The interaction of peptides with lipids was assessed by registering peptide-lipid contacts. It was assumed that the lipid interacts with the peptide if the distance between the peptide and lipid grains does not exceed the specified cutoff of $0.6 \mathrm{~nm}$. Such contacts were analyzed for each state obtained in the course of MD with a step of $0.1 \mathrm{~ns}$. The data obtained were displayed in two versions. In the first case, the total number of lipid molecules of each type interacting with the peptide was displayed. In the second case, maps of the intensity of contacts of amino acid residues of the peptide with cholesterol were constructed. These maps should show the areas with which cholesterol interacts over long periods of time, thus allowing determining cholesterol binding sites.

\section{RESULTS AND DISCUSSION}

Figure 1 illustrates the dependence of the number of DOPC (Figs. 1a-1d), DPSM (Figs. 1c, 1d), and cholesterol (Figs. 1b, 1d) molecules in contact with the peptide $\mathrm{p}_{1}$ on the MD time. It can be seen that the average number of contacting molecules is stabilized already after approximately $1 \mu$ s of the dynamics (Fig. 1c) and remains practically unchanged until the end of the calculated trajectory. The jump in the number of contacts of the peptide with lipids at $t \approx 1 \mu \mathrm{s}$ in Fig. $1 \mathrm{c}$ is due to the fact that the peptide was initially placed in the water near the membrane; when the peptide is inserted into the membrane at time moment $t \approx 1 \mu \mathrm{s}$, the number of peptide-lipid contacts increases sharply. All lipid molecules were distributed over the membrane almost homogeneously and did not form long-lived clusters among themselves (data not shown). The average number of DPSM and Chol molecules in contact with the peptide approximately corresponds to the concentration (mole fraction) of these lipids in the membrane. Indeed, in a singlecomponent membrane made of pure DOPC, on average, there are 10 molecules in contact with the peptide (Fig. 1a). In membranes made of the DOPC/DPSM and DOPC/Chol, the mole fraction of DPSM and Chol is $100 \% \times 20 /(180+20)=10 \%$. In these systems, on average, 10 molecules also contact the peptide, of which 9 are DOPC molecules and 1 DPSM (Fig. 1c) or Chol (Fig. 1b) molecule, in accordance with the mole fractions of these lipids. In a membrane of composition DOPC/DPSM/Chol 160/20/20, the mole fraction of DPSM and Chol is $10 \%$ each, and the mole fraction of DOPC is $80 \%$. Accordingly, in this system, on average, only 10 molecules are in contact with the $\mathrm{p}_{1}$ peptide, of which 8 molecules are DOPC, 1 molecule is DPSM, and 1 molecule is Chol (Fig. 1d). From the data obtained, it can be concluded that all modeled systems (DOPC; DOPC/Chol; DOPC/DPSM; DOPC/DPSM/Chol) are ideal in the sense that the $\mathrm{p}_{1}$ peptide does not have preferred (attraction) or undesirable (repulsion) interactions with any lipid. All contacts of the peptide with lipids are random, and their probability is proportional to the lipid mole fraction. If the peptide binds cholesterol to some extent, then the average number of contacts of the peptide with cholesterol should exceed the number of contacts proportional to the mole fraction of cholesterol in the membrane, provided that all lipids are approximately uniformly distributed laterally. For example, in the limiting case of a very strong long-lived bond of the peptide with one cholesterol molecule, the number of contacts should on average be approximately one more than in the case of an ideal system.

Figure 2 shows the similar time dependence of the number of contacting lipid molecules: DOPC 

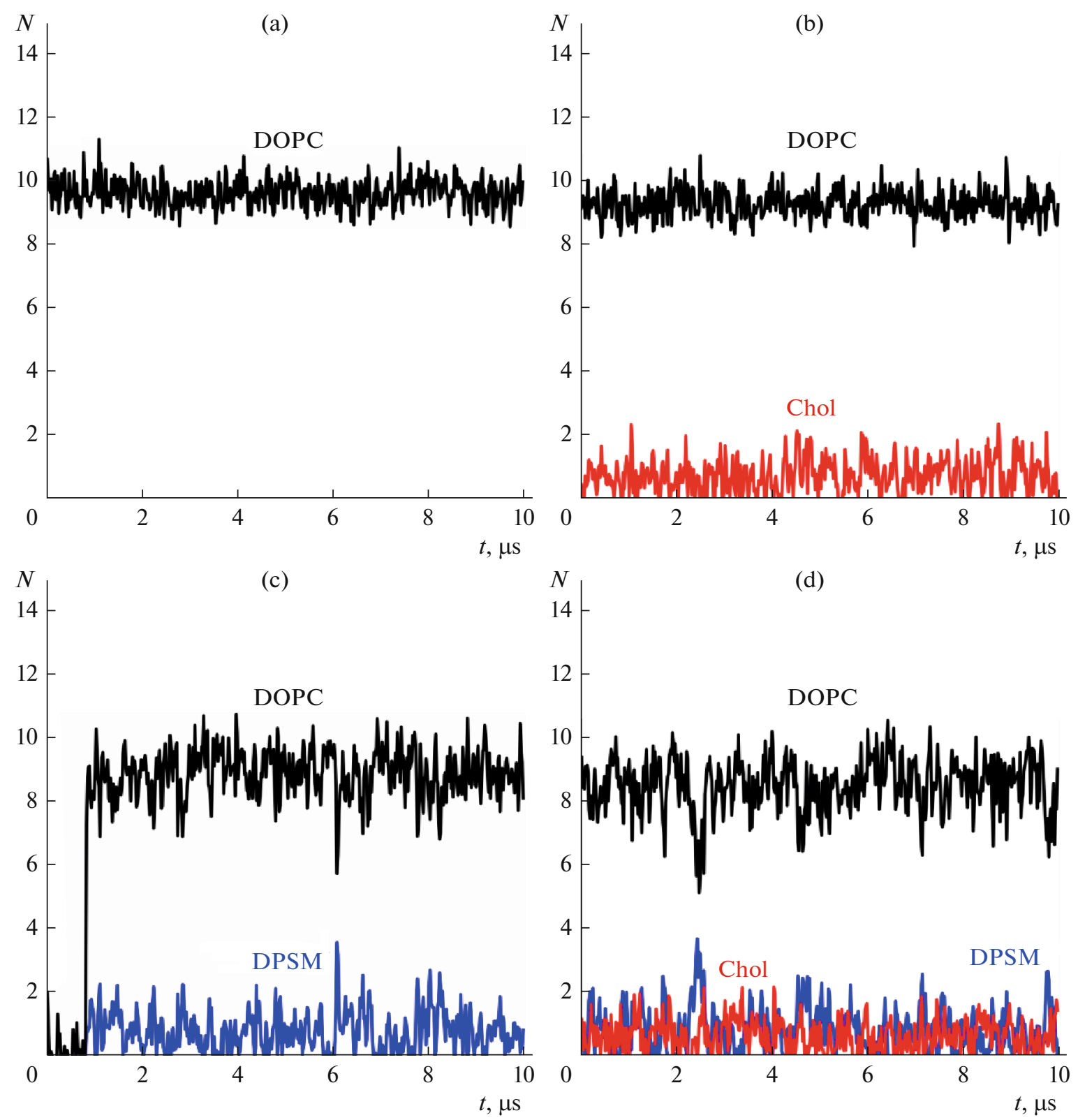

Fig. 1. Dependence of the number of lipid molecules in contact with peptide $\mathrm{p}_{1}$ on time in the membranes of the composition: (a) DOPC; (b) DOPC/Chol; (c) DOPC/DPSM; (d) DOPC/DPSM/Chol. The dependence of the number of DOPC molecules in contact with the peptide is shown in black; Chol, in red; DPSM, in blue.

(Figs. 2a-2d), DPSM (Figs. 2c, 2d), Chol (Figs. 2b, 2d) in membranes of different compositions. The jump in the number of contacts of the peptide with lipids at $t \approx 0.5 \mu \mathrm{s}$ in Fig. 2a and $t \approx 3 \mu \mathrm{s}$ in Fig. $2 \mathrm{c}$ is because the peptide was initially placed into the water near the membrane; the incorporation of the peptide into the membrane occurred at time moments $t \approx 0.5 \mu \mathrm{s}$ and $t \approx 3 \mu \mathrm{s}$, respectively, and the number of peptide-lipid contacts with the basic lipid (DOPC) increased sharply. Peptide $\mathrm{p}_{2}$ is larger (consists of more amino acid residues) than peptide $\mathrm{p}_{1}$; accordingly, in a membrane made of pure DOPC, on average, about 12 DOPC molecules are in contact with the peptide $\mathrm{p}_{2}$ (compare Figs. 2a and 1a). As in the case of the peptide $\mathrm{p}_{1}$, the simulated systems "membrane + peptide $\mathrm{p}_{2}$ " are ideal in the sense that the average number of lipid molecules in contact with the peptide is proportional to their mole fraction. Indeed, in the membrane of the composition DOPC/Chol 180/20, the mole fraction of cholesterol is $10 \%$; in such a membrane, the $\mathrm{p}_{2}$ peptide contacts, on average, approximately 11 DOPC molecules and 1 cholesterol molecule (Fig. 2b). In the DOPC/DPSM $180 / 20$ membrane, the mole fraction of DPSM is also $10 \%$, and the $\mathrm{p}_{2}$ peptide is in contact on average with 

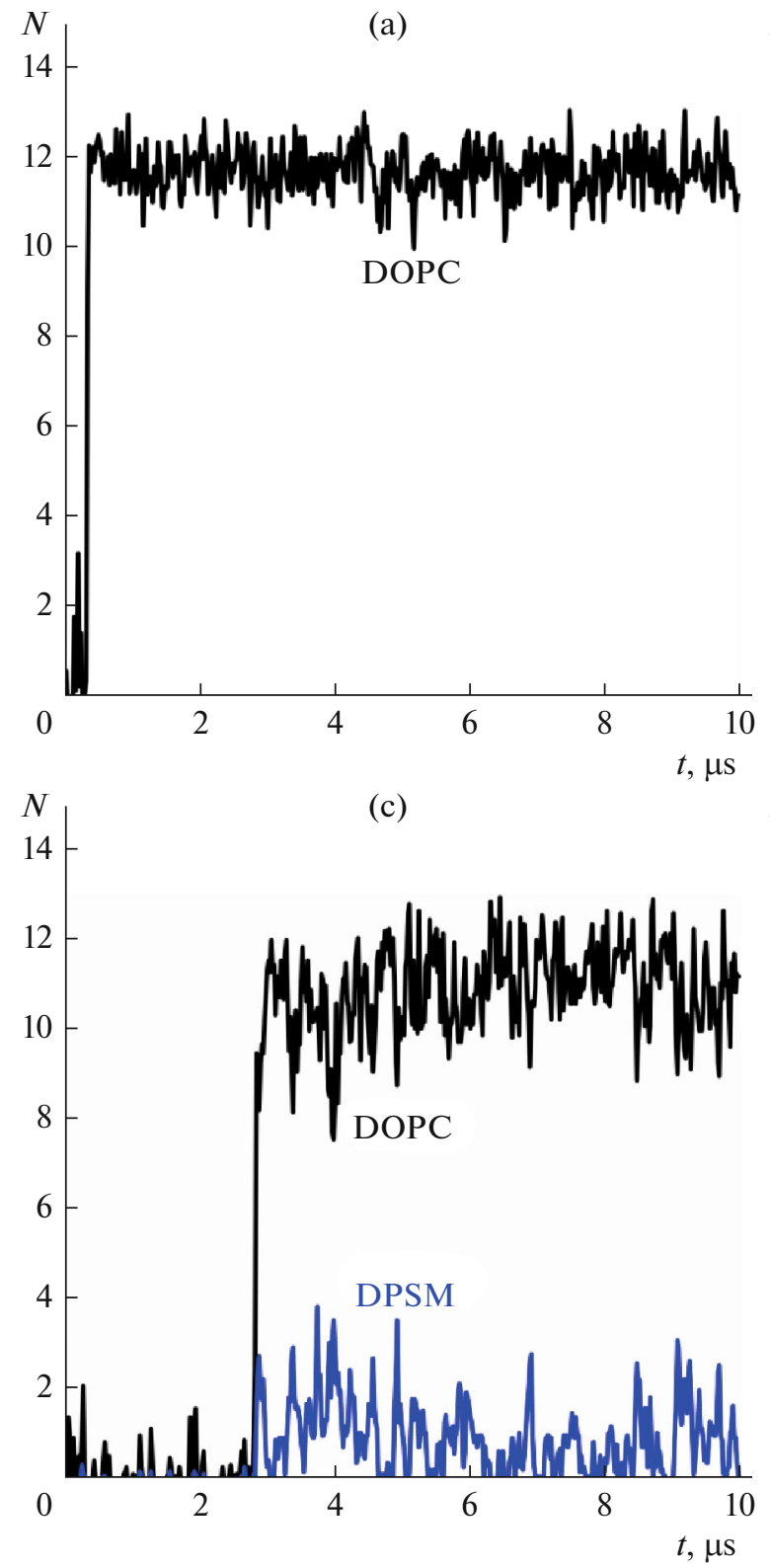

(b)

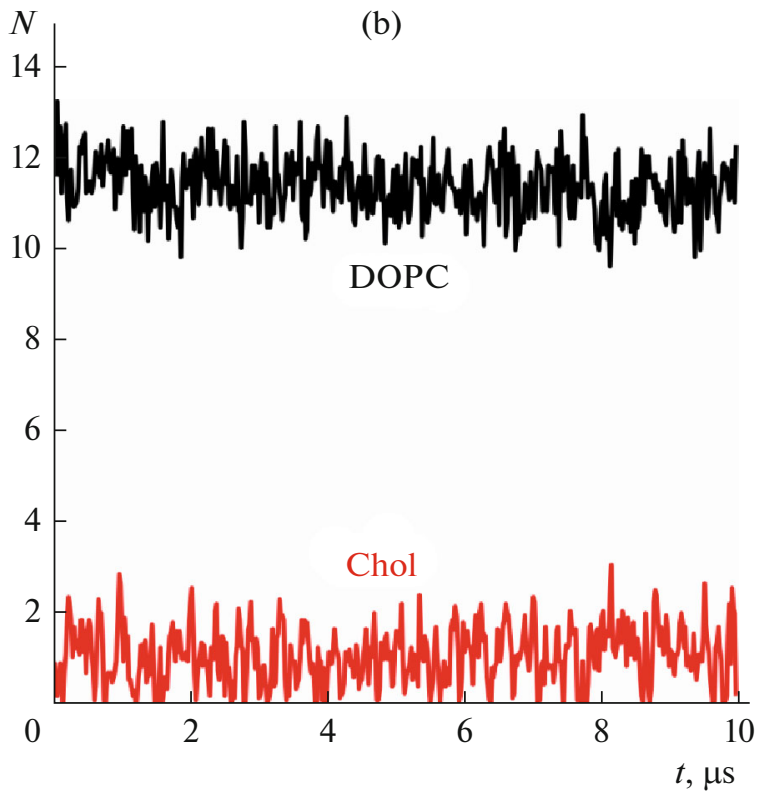

(d)

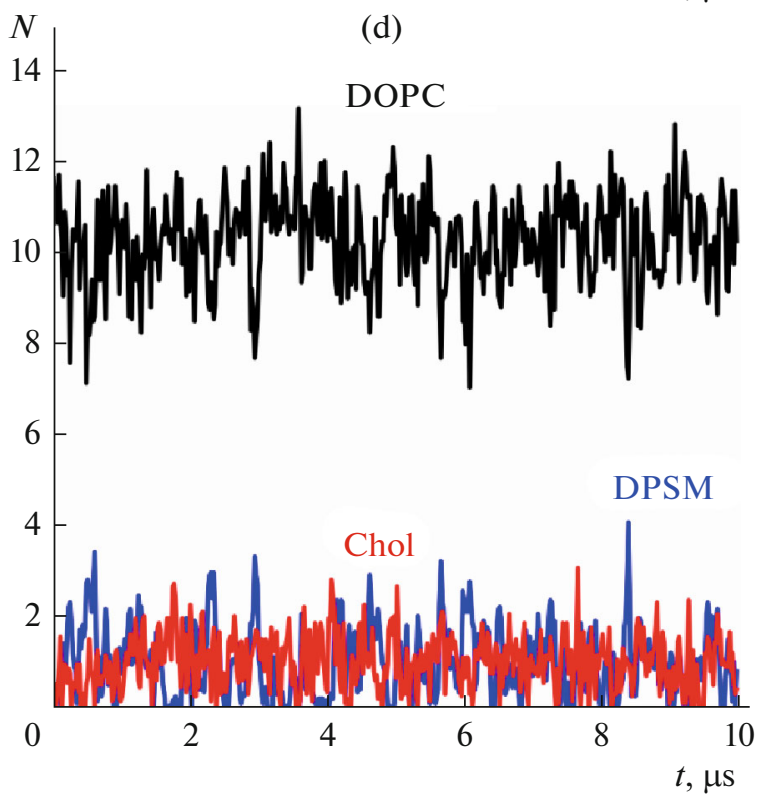

Fig. 2. Dependence of the number of lipid molecules in contact with peptide $\mathrm{p}_{2}$ on time in the membranes of the composition: (a) DOPC; (b) DOPC/Chol; (c) DOPC/DPSM; (d) DOPC/DPSM/Chol. The dependence of the number of DOPC molecules in contact with the peptide is shown in black; Chol, in red; DPSM, in blue.

about 11 DOPC molecules and 1 DPSM molecule (Fig. 2c).

In the DOPC/DPSM/Chol 160/20/20 membrane, the mole fractions of the components are 80, 10 , and $10 \%$ for DOPC, DPSM, Chol, respectively. In this case, about 10 DOPC molecules, 1 DPSM molecule, and 1 cholesterol molecule are in contact with the $\mathrm{p}_{2}$ peptide on average, in proportion to the mole fraction of each lipid component in the membrane (Fig. 2d). Thus, the peptide $\mathrm{p}_{2}$ has neither preferred (attraction) nor undesirable (repulsion) interactions with any of the lipids.
Figure 3 shows diagrams of the time dependence of cholesterol contacts with amino acid residues of peptides $\mathrm{p}_{1}$ (Figs. 3a, 3b) and $\mathrm{p}_{2}$ (Figs. 3c, 3d) in membranes of compositions DOPC/Chol (Figs. 3a, 3c) and DOPC/DPSM/Chol (Figs. 3b, 3d). The contact of any amino acid residue with cholesterol at a given time moment is indicated by a vertical gray stripe on the diagrams, the absence of contact is indicated by a vertical white stripe.

Figure 3 shows that cholesterol interacts predominantly with hydrophobic amino acid residues oriented towards the membrane interior. In this case, none of 
(a)

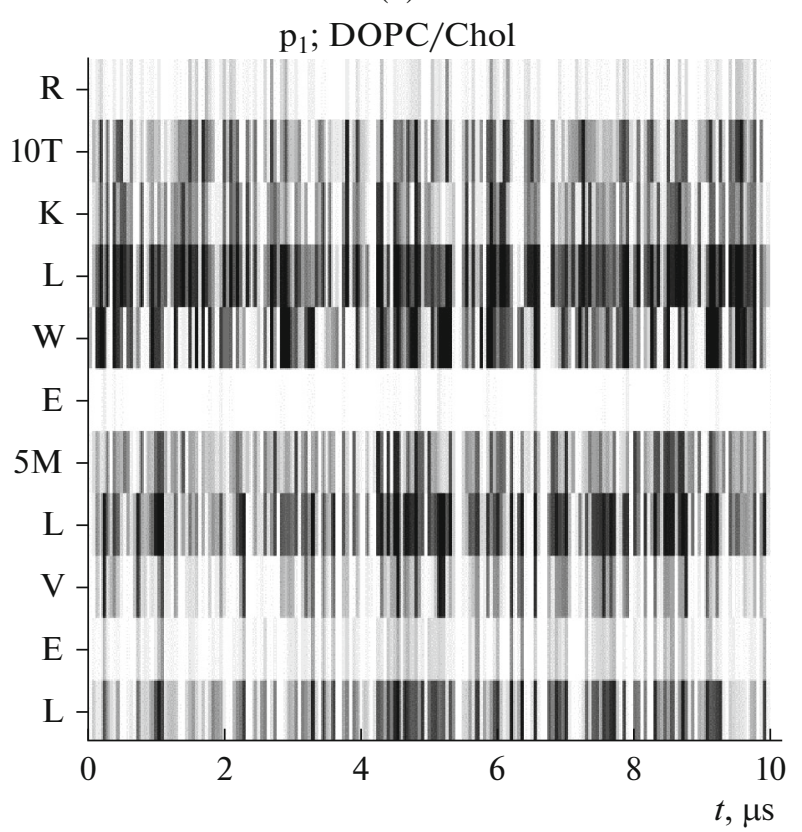

(c)

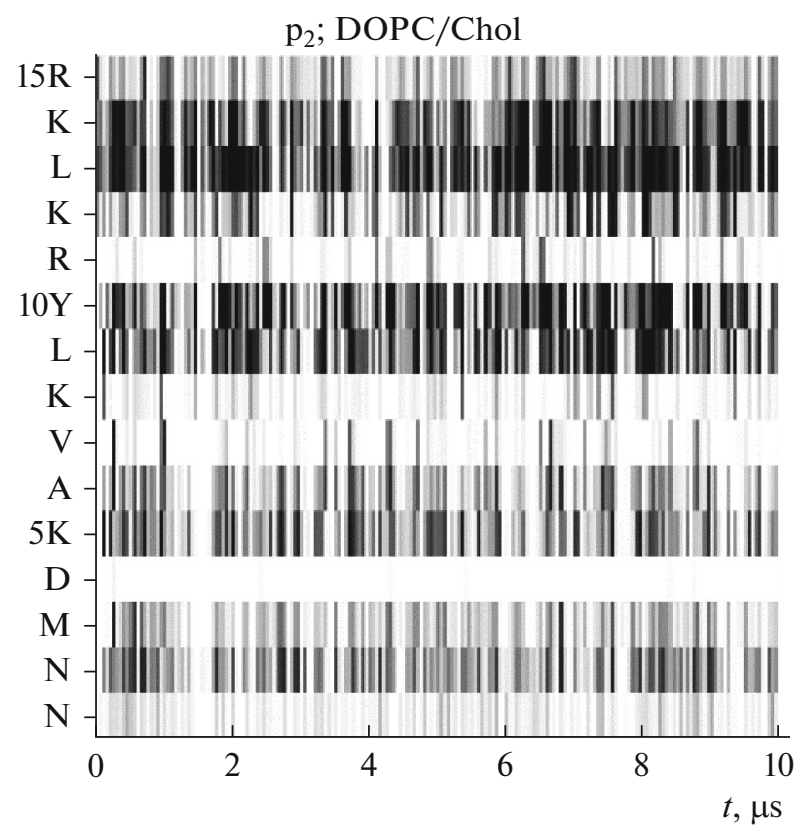

(b)

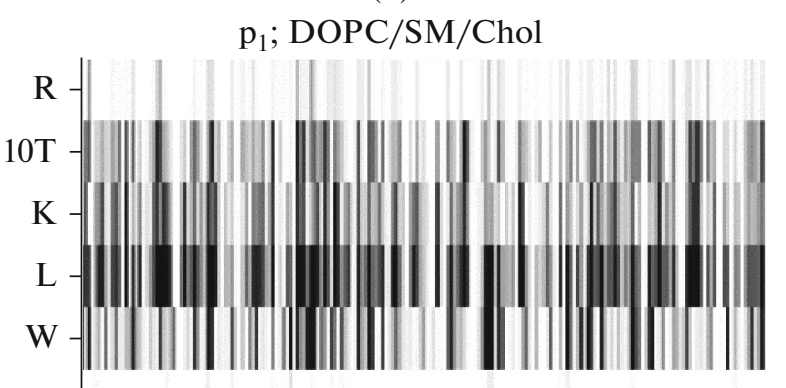

E -

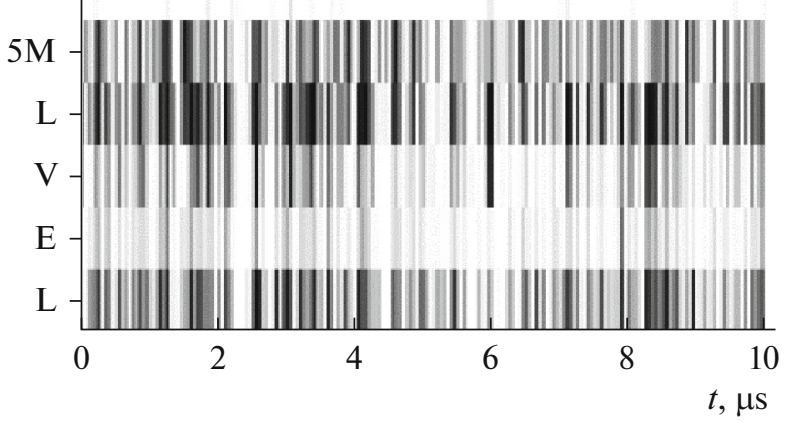

(d)

$\mathrm{p}_{2} ; \mathrm{DOPC} / \mathrm{SM} / \mathrm{Chol}$

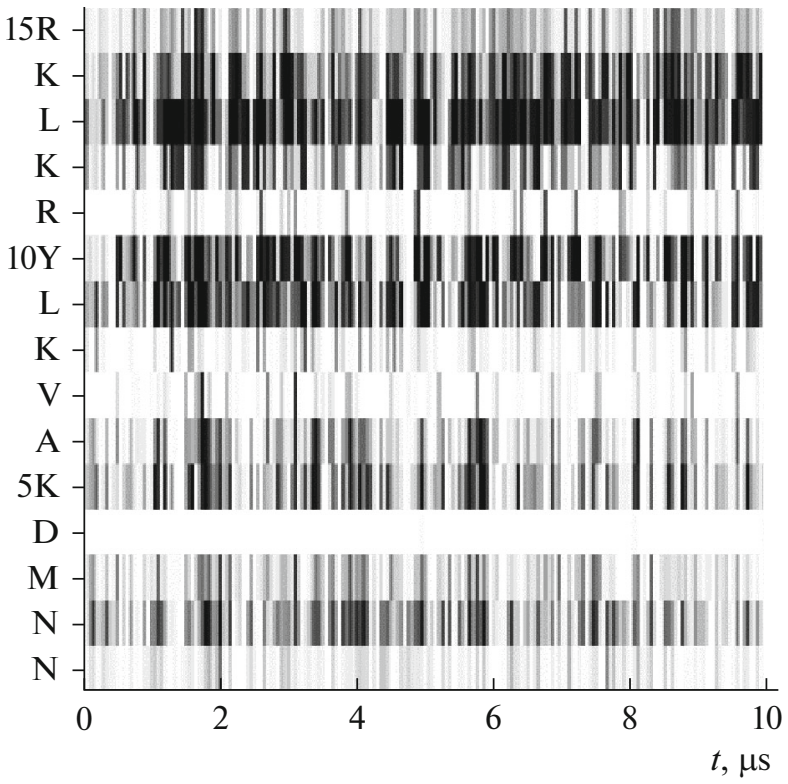

Fig. 3. Diagrams of the time dependence of cholesterol contacts with amino acid residues of peptides $p_{1}(a, b)$ and $p_{2}(c, d)$ in membranes of composition DOPC/Chol (a, c) and DOPC/DPSM/Chol (b, d). The contact of an amino acid residue with cholesterol at a given time moment is indicated by a vertical gray stripe in the diagrams, the absence of the contact is indicated by a vertical white stripe.

the peptides has stable cholesterol binding sites and does not form long-lived complexes with cholesterol. From the fact that the characteristic time of continuous presence of a cholesterol molecule near specific amino acid residues of peptides is relatively short $(<0.4 \mu \mathrm{s}$, see Fig. 3), and the average number of cholesterol molecules in contact with peptides is proportional to the mole fraction of cholesterol, it can be concluded that the interactions of peptide-cholesterol are random. From the obtained MD results, it follows that the presence of CRAC motif in the peptide structure is not a sufficient condition for peptide's preferential interaction with cholesterol.

How membrane proteins are sorted between the $\mathrm{L}_{\mathrm{o}}$ and $\mathrm{L}_{\mathrm{d}}$ phases of the membrane is currently not fully understood. In a number of works [35-40], the lateral 
distribution of the LAT (Linker for Activation of T-cells) transmembrane protein and its various derivatives and mutants are systematically studied. It is found [39] that the preference for the ordered or disordered phase by transmembrane proteins is determined by three features of their chemical structure: (1) posttranslational modification of proteins (attachment of palmitic or myristic acid residues) increases the distribution coefficient in the $\mathrm{L}_{\mathrm{o}}$ phase; (2) so-called accessible surface area of the transmembrane domain (TMD) of a protein, which is determined by the volume of its constituent amino acids and correlates with the TMD diameter (a smaller diameter promotes distribution into the $\mathrm{L}_{\mathrm{o}}$ phase); (3) TMD length-an increase in length by one amino acid relatively increases the distribution coefficient into the $\mathrm{L}_{\mathrm{o}}$ phase by about $5 \%$ [40]. It is shown that such changes in the TMD protein structure lead to approximately additive changes in the distribution coefficient of proteins between membrane phases. By molecular dynamics methods, it is demonstrated that LAT has a strong preference for the $\mathrm{L}_{\mathrm{o}} / \mathrm{L}_{\mathrm{d}}$ interface [38]. The calculated dependencies of the energy (potential of mean force) on the position of the transmembrane protein relative to the interface are in good agreement with the analogous dependencies of the energy obtained by methods of the continuum theory of elasticity [41, 42]. However, the data of works [35-40] do not allow explaining the distribution of peripheral proteins between membrane phases.

The chemical structure of CRAC motifs is determined by the general formula: -L/V-(X)(1-5)-Y/W$(\mathrm{X})(1-5)-\mathrm{R} / \mathrm{K}-$, where $(\mathrm{X})(1-5)$ denotes from one to five residues of any amino acids, the symbol "/" means "or". Thus, any CRAC motif is amphipathic, since it contains simultaneously charged $(\mathrm{R} / \mathrm{K})$, aromatic $(\mathrm{Y} / \mathrm{W})$, and hydrophobic $(\mathrm{L} / \mathrm{V})$ amino acid residues. In [41, 42], the predominant distribution of various membrane inclusions between the bulk $\mathrm{L}_{\mathrm{o}}$ and $\mathrm{L}_{\mathrm{d}}$ phases and the interface is theoretically studied. It is shown that the predominant distribution into the region of the $L_{o} / L_{d}$ interface is characteristic for (i) all lipid inclusions, the monolayer of which has a nonzero spontaneous curvature; (ii) all peripheral membrane inclusions, both amphipathic and predominantly hydrophobic; (iii) transmembrane inclusions that have a non-cylindrical shape, for example, "hourglass" or "barrel". The distribution of such membrane inclusions to the domain boundary always leads to a decrease in the boundary energy. Only lipid inclusions, monolayers of which have approximately zero spontaneous curvature, had no preference for the interface. Besides, cylindrical transmembrane inclusions, the length of which approximately coincided with the thickness of the lipid bilayer of the corresponding phase, preferred to be distributed into the bulk $\mathrm{L}_{\mathrm{o}}$ or $\mathrm{L}_{\mathrm{d}}$ phase [41]. Thus, basing on the results of [41, 42], any peripheral peptide including an amphipathic CRAC motif should have a strong preference for the $\mathrm{L}_{\mathrm{o}} / \mathrm{L}_{\mathrm{d}}$ interface. For this, the peptide does not need to bind cholesterol, since the driving force of lateral redistribution is the interaction of peptideinduced membrane deformations caused by the amphipathic nature of the peptide and membrane deformations occurring at the $\mathrm{L}_{\mathrm{o}} / \mathrm{L}_{\mathrm{d}}$ interface $[42,43]$. In the transmembrane peptide, apparently, there should be a tendency to the location of the CRAC motif near the outer (polar) boundaries of the membrane, since the motif includes positively charged amino acids $(\mathrm{R} / \mathrm{K})$. The characteristic amino acids of the CRAC motifs are quite bulky. The presence of bulky amino acids in the composition of the transmembrane region near the membrane surfaces can provide an effective "hourglass" shape of the transmembrane peptide: a large volume near the hydrophilic surfaces of the membrane and a small one in the area of the intermonolayer surface. According to the results of work [41], the optimal position of the transmembrane peptide, which has an effective "hourglass" shape, is near the boundary of the $\mathrm{L}_{\mathrm{o}}$ and $\mathrm{L}_{\mathrm{d}}$ phases.

It should be noted that all raft-dependent processes are automatically cholesterol-dependent, but the opposite is not true. According to theoretical results of works [41, 42], amphipathic peptides should be predominantly distributed to the raft boundary, change the boundary energy, and affect the properties of the raft ensemble. However, the amphipathic nature of the peptide is not a sufficient condition for its effect on cholesterol-dependent processes. It was shown in [26] that a peptide obtained by combining peptides $\mathrm{p}_{1}$ and $\mathrm{p}_{2}$ has a strong and complex effect on the cholesteroldependent activity of cells. When aromatic amino acids in two CRAC motifs are replaced with serine, the effect of the peptide practically disappears [44], although the replacement of aromatic amino acids with serine does not change the amphipathic properties of the peptide.

In [45], the interaction with membranes of different composition of two peptides containing CRAC motifs is studied; the peptides are parts of leukotoxin LtxA. The study is carried out by methods of all-atom molecular dynamics. A comparative analysis of the binding of peptides and changes in their secondary structure with membranes consisting of pure dimyristoylphosphatidylcholine (DMPC) and a mixture of DMPC/Chol $60: 40$ are carried out. It is shown that both peptides are embedded deeper into membranes of the composition DMPC/Chol $60: 40$ than into membranes from pure DMPC. Upon insertion, the secondary structure of peptides changes, and these changes are also more pronounced in the membranes of composition DMPC/Chol $60: 40$. However, in this work, the binding (number of contacts) of CRAC motifs with cholesterol is not directly analyzed. In principle, at least partially, the observed effects can be explained by a change in the integral properties of the membrane upon addition of cholesterol, rather than 
by the specific interaction of CRAC motifs with cholesterol. The incorporation of an amphipathic peptide into the membrane inevitably leads to its deformation. The energy of the resulting deformations, in turn, should affect the efficiency of incorporation and, possibly, the final conformation of the peptide. For example, in the limiting case of a very rigid membrane, the deformation energy can be so great that the peptide cannot incorporate into the membrane at all. It is known that cholesterol affects the thickness and bending rigidity of the membrane, as well as the spontaneous curvature of its monolayers [13, 14, 46-48]. These parameters determine the energy of deformations occurring near the amphipathic peptide embedded into the membrane, and, thus, can influence the depth of incorporation. In this case, the interaction of the CRAC containing peptide with the membrane can be determined by membrane's integral properties, rather than by the efficiency or specificity of binding of the peptide to cholesterol; however, it is not a trivial task to reliably distinguish between these two mechanisms.

In [49], sites of preferential interaction with cholesterol of the serotonin receptor are analyzed using the coarse-grained MD method. There are seven transmembrane regions and three CRAC motifs in the structure of the receptor. It is found that cholesterol interacts to a comparable extent with all transmembrane helices of the receptor, and even the identified sites of preferential interaction contact with cholesterol molecules for a time comparable to the time of occupation of other regions of the transmembrane helices. In this case, cholesterol repeatedly binds (comes into contact) with such sites and dissociates from them during the calculated MD trajectory. An increased time of contact with cholesterol is manifested in only one of three CRAC motifs, and this time was not the maximum one among the identified sites of preferential interaction with cholesterol. The characteristic time of interaction of transmembrane helices with palmitoyleoylphosphatidylcholine (POPC) in the membrane of composition POPC/Chol 50:50 turned out to be comparable with the time of interaction with cholesterol, and the binding of a particular site with POPC did not exclude its simultaneous binding with cholesterol. Thus, it is shown that the selectivity of the identified sites of predominant interaction with cholesterol is quite low.

In [50], the interaction with the membranes of the serotonin receptor is modeled using the coarsegrained MD methods. 41 sites of preferential contacts with cholesterol are found, of which only two belong to CRAC motifs. The number of contacts of each site with cholesterol correlated with its steric availability for cholesterol, i.e., with the degree of site immersion into the hydrophobic part of the membrane and its orientation. For one of the two sites belonging to CRAC motifs, the selectivity for cholesterol binding is quite low.
Thus, from the results of our calculations and available literature data, we can conclude that the presence of CRAC motif in a primary structure of protein or peptide is not a universal criterion for its preferential interaction or binding with cholesterol: CRAC containing peptides or protein regions can either bind or not bind cholesterol; in addition, cholesterol can also bind to peptides or protein regions not containing CRAC motifs [45, 49, 50]. The predominant distribution of proteins containing CRAC motifs in their structure into the liquid-ordered phase of the membrane or on its boundary can be determined by the amphipathic nature of this amino acid sequence, which provides a certain molecular geometry of peptides [35-40]. The strong interaction of peptides containing CRAC motifs with membranes containing cholesterol, in addition to the specific interaction of the CRAC motif with cholesterol, can also be explained by the strong influence of cholesterol on the integral physicochemical properties of the membrane, on which the character of interactions of the peptide with the membrane depends $[41,42,51]$.

\section{ACKNOWLEDGMENTS}

This work was supported by the Ministry of Science and Higher Education of the Russian Federation, Russian Foundation for Basic Research (project nos. 18-04-01363 and 20-54-00041), and partially by the grant of the President of the Russian Federation (MK-3119.2019.4).

\section{COMPLIANCE WITH ETHICAL STANDARDS}

The authors declare that they have no conflict of interest.

This article does not contain any studies involving animals or human participants performed by any of the authors.

\section{OPEN ACCESS}

This article is licensed under a Creative Commons Attribution 4.0 International License, which permits use, sharing, adaptation, distribution and reproduction in any medium or format, as long as you give appropriate credit to the original author(s) and the source, provide a link to the Creative Commons license, and indicate if changes were made. The images or other third party material in this article are included in the article's Creative Commons license, unless indicated otherwise in a credit line to the material. If material is not included in the article's Creative Commons license and your intended use is not permitted by statutory regulation or exceeds the permitted use, you will need to obtain permission directly from the copyright holder. To view a copy of this license, visit http://creativecommons.org/licenses/by/4.0/.

\section{REFERENCES}

1. Lillemeier B.F., Pfeiffer J.R., Surviladze Z., Wilson B.S., Davis M.M. 2006. Plasma membrane-associated pro- 
teins are clustered into islands attached to the cytoskeleton. Proc. Natl. Acad. Sci. USA. 103, 18992-18997.

2. Simons K., Ikonen, E. 1997. Functional rafts in cell membranes. Nature. 387, 569-572.

3. Ayuyan A.G., Cohen F.S. 2008. Raft composition at physiological temperature and $\mathrm{pH}$ in the absence of detergents. Biophys. J. 94, 2654-2666.

4. Edidin M. 2001. Shrinking patches and slippery rafts: Scales of domains in the plasma membrane. Trends Cell Biol. 11, 492-496.

5. Veatch S.L., Polozov I.V., Gawrisch K., Keller S.L. 2004. Liquid domains in vesicles investigated by NMR and fluorescence microscopy. Biophys. J. 86, 29102922.

6. Pike L.J. 2006. Rafts defined: A report on the Keystone Symposium on lipid rafts and cell function. J. Lip. Res. 47, 1597-1598.

7. Yang S.T., Kiessling V., Simmons J.A., White J.M., Tamm L.K. 2015. HIV gp41-mediated membrane fusion occurs at edges of cholesterol-rich lipid domains. Nat. Chem. Biol. 11, 424-431.

8. Allen J.A., Halverson-Tamboli R.A., Rasenick M.M. 2007. Lipid raft microdomains and neurotransmitter signalling. Nat. Rev. Neurosci. 8, 128-140.

9. Staneva G., Osipenko D.S., Galimzyanov T.R., Pavlov K.V., Akimov S.A. 2016. Metabolic precursor of cholesterol causes formation of chained aggregates of liquid-ordered domains. Langmuir. 32, 1591-1600.

10. Saitov A., Akimov S.A., Galimzyanov T.R., Glasnov T., Pohl P. 2020. Ordered lipid domains assemble via concerted recruitment of constituents from both membrane leaflets. Phys. Rev. Lett. 124, 108102.

11. Baumgart T., Hess S.T., Webb W.W. 2003. Imaging coexisting fluid domains in biomembrane models coupling curvature and line tension. Nature. 425, 821-824.

12. Samsonov A.V., Mihalyov I., Cohen F.S. 2001. Characterization of cholesterol-sphingomyelin domains and their dynamics in bilayer membranes. Biophys. J. 81, 1486-1500.

13. Rinia H.A., Snel M.M., van der Eerden J.P., de Kruijff B. 2001. Visualizing detergent resistant domains in model membranes with atomic force microscopy. FEBS Lett. 501, 92-96.

14. Baumgart T., Das S., Webb W.W., Jenkins J.T. 2005. Membrane elasticity in giant vesicles with fluid phase coexistence. Biophys. J. 89, 1067-1080.

15. Akimov S.A., Molotkovsky R.J., Kuzmin P.I., Galimzyanov T.R., Batishchev O.V. 2020. Continuum models of membrane fusion: Evolution of the theory. Int. J. Mol. Sci. 21, 3875.

16. Espadas J., Pendin D., Bocanegra R., Escalada A., Misticoni G., Trevisan T., del Olmo A.V., Montagna A., Bova S., Ibarra B., Kuzmin P.I., Bashkirov P.V., Shnyrova A.V., Frolov V.A., Daga A. 2019. Dynamic constriction and fission of endoplasmic reticulum membranes by reticulon. Nat. Comm. 10, 5327.

17. Bocharov E.V., Mineev K.S., Pavlov K.V., Akimov S.A., Kuznetsov A.S., Efremov R.G., Arseniev A.S. 2017. Helix-helix interactions in membrane domains of bitopic proteins: Specificity and role of lipid environment. Biochim. Biophys. Acta. 1859, 561-576.
18. Doronin I.I., Vishnyakova P.A., Kholodenko I.V., Ponomarev E.D., Ryazantsev D.Y., Molotkovskaya I.M., Kholodenko R.V. 2014. Ganglioside GD2 in reception and transduction of cell death signal in tumor cells. BMC Cancer. 14, 295.

19. Polozov I.V., Bezrukov L., Gawrisch K., Zimmerberg J. 2008. Progressive ordering with decreasing temperature of the phospholipids of influenza virus. Nat. Chem. Biol. 4, 248-255.

20. Epand R.M. 2008. Proteins and cholesterol-rich domains. Biochim. Biophys. Acta. 1778, 1576-1582.

21. Li H., Papadopoulos V. 1998. Peripheral-type benzodiazepine receptor function in cholesterol transport. Identification of a putative cholesterol recognition/interaction amino acid sequence and consensus pattern. Endocrinology. 139, 4991-4997.

22. Epand R.M., Sayer B.G., Epand R.F. 2005. Caveolin scaffolding region and cholesterol-rich domains in membranes. J. Mol. Biol. 345, 339-350.

23. de Vries M., Herrmann A., Veit M. 2015. A cholesterol consensus motif is required for efficient intracellular transport and raft association of a group 2 HA from influenza virus. Biochem. J. 465, 305-314.

24. Tsfasman T., Kost V., Markushin S., Lotte V., Koptiaeva I., Bogacheva E., Baratova L., Radyukhin V. 2015. Amphipathic alpha helices and putative cholesterol binding domains of the influenza virus matrix M1 protein are crucial for virion structure organization. Virus Res. 210, 114-118.

25. Dunina-Barkovskaya A.Y., Vishnyakova K.S., Golovko A.O., Arutyunyan A.M., Baratova L.A., Bathishchev O.V., Radyukhin V.A. 2018. Amphipathic CRAC-containing peptides derived from the influenza virus A M1 protein modulate cholesterol-dependent activity of cultured IC-21 macrophages. Biochemistry (Moscow). 83, 982-991.

26. Dunina-Barkovskaya A.Y., Vishnyakova K.S., Baratova L.A., Radyukhin V.A. 2019. Modulation of cholesterol-dependent activity of macrophages IC-21 by a peptide containing two CRAC motifs from protein M1 of influenza virus. Biol. Membrany (Rus.). 36, 271-280.

27. Frisz J.F., Klitzing H.A., Lou K., Hutcheon I.D., Weber P.K., Zimmerberg J., Kraft M.L. 2013. Sphingolipid domains in the plasma membranes of fibroblasts are not enriched with cholesterol. J. Biol. Chem. 288, 16855-16861.

28. de Jong D.H., Singh G., Drew Bennett W.F., Arnarez C., Wassenaar T.A., Schäfer L.V., Periole X., Tieleman D.P., Marrink S.J. 2013. Improved parameters for the MARTINI coarse-grained protein force field. J. Chem. Theor. Comp. 9, 687-697.

29. Marrink S.J., Risselada H.J., Yefimov S., Tieleman D.P., de Vries A.H. 2007. The MARTINI force field: Coarse grained model for biomolecular simulations. J. Phys. Chem. B 111, 7812-7824.

30. Nosé S. 1984. A molecular dynamics method for simulations in the canonical ensemble. Mol. Phys. 52, 255268.

31. Hoover W.G. 1985. Canonical dynamics: Equilibrium phase-space distributions. Phys. Rev. A. 31, 1695-1697. 
32. Parrinello M., Rahman A. 1981. Polymorphic transitions in single crystals: A new molecular dynamics method. J. Appl. Phys. 52, 7182-7190.

33. Nosé S., Klein M.L. 1983. Constant pressure molecular dynamics for molecular systems. Mol. Phys. 50, 10551076.

34. Berendsen H.J.C., Postma J.P.M., DiNola A., Haak J.R. 1984. Molecular dynamics with coupling to an external bath. J. Chem. Phys. 81, 3684-3690.

35. Levental I., Lingwood D., Grzybek M., Coskun U., Simons K. 2010. Palmitoylation regulates raft affinity for the majority of integral raft proteins. Proc. Natl. Acad. Sci. USA. 107, 22050-22054.

36. Lorent J.H., Levental I. 2015. Structural determinants of protein partitioning into ordered membrane domains and lipid rafts. Chem. Phys. Lipids. 192, 23-32.

37. Levental I., Grzybek M., Simons K. 2010. Greasing their way: Lipid modifications determine protein association with membrane rafts. Biochemistry. 49, 63056316.

38. Lin X., Gorfe A.A., Levental I. 2018. Protein partitioning into ordered membrane domains: Insights from simulations. Biophys. J. 114, 1936-1944.

39. Lorent J.H., Diaz-Rohrer B., Lin X., Spring K., Gorfe A.A., Levental K.R., Levental I. 2017. Structural determinants and functional consequences of protein affinity for membrane rafts. Nat. Commun. 8, 1219.

40. Diaz-Rohrer B.B., Levental K.R., Simons K., Levental I.2014. Membrane raft association is a determinant of plasma membrane localization. Proc. Natl. Acad. Sci. USA. 111, 8500-8505.

41. Pinigin K.V., Kondrashov O.V., Jiménez-Munguía I., Alexandrova V.V., Batishchev O.V., Galimzyanov T.R., Akimov S.A. 2020. Elastic deformations mediate interaction of the raft boundary with membrane inclusions leading to their effective lateral sorting. Sci. Rep. 10, 4087.

42. Pinigin K.V., Volovik M.V., Batishchev O.V., Akimov S.A. 2020. Interaction of ordered lipid domain boundaries and amphipathic peptides regulates probability of pore formation in membranes. Biol. Membrany (Rus.). 37, 337-349.

43. Galimzyanov T.R., Molotkovsky R.J., Kuzmin P.I., Akimov S.A. 2011. Stabilization of the raft bilayer structure due to elastic deformations of the membrane. Biol. Membrany (Rus.). 28, 307-314.

44. Dunina-Barkovskaya A.Y., Vishnyakova K.S. 2020. Modulation of the cholesterol-dependent activity of macrophages IC-21 by CRAC peptides with substituted motive-forming amino acids. Biol. Membrany (Rus.). 37, 381-395.

45. Miller C.M., Brown A.C., Mittal J. 2014. Disorder in cholesterol-binding functionality of CRAC peptides: A molecular dynamics study. J. Phys. Chem. B. 118, 13169-13174.

46. Evans E., Rawicz W. 1990. Entropy-driven tension and bending elasticity in condensed-fluid membranes. Phys. Rev. Lett. 64, 2094.

47. Gandhavadi M., Allende D., Vidal A., Simon S.A., McIntosh T.J. 2002. Structure, composition, and peptide binding properties of detergent soluble bilayers and detergent resistant rafts. Biophys. J. 82, 1469-1482.

48. Kollmitzer B., Heftberger P., Rappolt M., Pabst G. 2013. Monolayer spontaneous curvature of raft-forming membrane lipids. Soft Matter. 9, 10877-10884.

49. Sengupta D., Chattopadhyay A. 2012. Identification of cholesterol binding sites in the serotonin1A receptor. J. Phys. Chem. B. 116, 12991-12996.

50. Ferraro M., Masetti M., Recanatini M., Cavalli A., Bottegoni G. 2016. Mapping cholesterol interaction sites on serotonin transporter through coarse-grained molecular dynamics. PloS ONE. 11, e0166196.

51. Karpunin D.V., Akimov S.A., Frolov V.A. 2005. Pore formation in lipid membranes containing lysolipids and cholesterol. Biol. Membrany (Rus.). 22, 429-432.

Translated by S. Akimov 